\title{
A Qualitative Study on the Effects of Mesh Guideline Modification for Unstructured Mesh Generation of the NASA High Lift Common Research Model (HL-CRM)
}

\author{
William T. Jones, ${ }^{*}$ \\ NASA Langley Research Center, Hampton, VA 23681-2199 \\ Carolyn Woeber ${ }^{\dagger}$ \\ Pointwise, Inc., Fort Worth, TX 76104
}

\begin{abstract}
As part of the $1^{\text {st }}$ Geometry and Mesh Generation Workshop, unstructured tetrahedral and unstructured hybrid Computational Fluid Dynamics meshes were generated according to the meshing guidelines supplied by the $3^{\text {rd }}$ High Lift Prediction Workshop. During this process, it was noted that application of some meshing guidelines became a bottleneck in the process and negatively impacted the quality of the meshes. A study is performed to compare the FUN3D simulation from the baseline medium-resolution workshop unstructured mesh with those on meshes resulting from guideline variations to the baseline. Recommendations on the elimination or reduction of meshing guidelines for high lift aerodynamic cases like the High Lift Common Research Model are based on the resulting data.
\end{abstract}

\section{Nomenclature}

$\mathrm{C}_{\mathrm{L}} \quad$ lift coefficient

M Mach number

$\mathbf{R}_{\mathbf{e}} \quad$ Reynolds number
Symbols

$\alpha \quad$ angle of attack

$\mathcal{O} \quad$ asymptotic algorithm complexity, i.e., big $O$ notation

\section{Introduction}

$\prod$

HE $1^{\text {st }}$ Geometry and Mesh Generation Workshop (GMGW1), held in Denver, CO in June 2017 and 1 sponsored by the AIAA Meshing, Visualization, and Computational Environments Technical Committee, focused on documenting the mesh generation process, analyzing the resulting meshes, and downselecting to provide baseline meshes for the High Lift Common Research Model ${ }^{1}$ (HL-CRM) geometry. The $3^{\text {rd }}$ High Lift Prediction Workshop (HLPW3), sponsored by the AIAA Applied Aerodynamics Technical Committee, was held concurrently and supplied the meshing guidelines ${ }^{2}$ used by the GMGW1 committee members and participants to generate structured multiblock, unstructured, hybrid, and overset mesh families. During the course of the GMGW1, committee members and participants identified specific meshing guidelines that were difficult to comply with, made mesh generation slower, or generated what they felt were suboptimal quality meshes. $^{3-5}$

The impetus for this study came from these mesh generation practitioners advocating for the review and possible elimination of specific guidelines during the workshop. Assessing the effect of eliminating a guideline, or expanding the range of effective values for a guideline, within the current set of established best practices, could enable a reduction in the time and resources involved for mesh generation of a high lift configuration such as the HL-CRM. Note that the reduction in time and resource from this type of guideline modification is currently dependent on the specific guideline and its impact on the workflow for a particular mesh generation

\footnotetext{
${ }^{*}$ Computer Engineer, Computational AeroSciences Branch, MS 128, AIAA Associate Fellow

${ }^{\dagger}$ Manager, Technical Support Team, AIAA Senior Member
} 
tool. Here instead, establishment of which guideline modifications/eliminations have minimal impact on the resulting CFD simulations is the primary focus instead of how those modifications affect the time to mesh and resources involved. Evaluating the effect on time to mesh and resource usage will be the focus of future work. Specific guidelines will be evaluated by modifying the medium-resolution baseline mesh provided by the GMGW1 committee and constructed with the Pointwise ${ }^{\circledR}$ mesh generation software. Solutions will be run on those mesh variants using the FUN3D unstructured, node-based, second-order, finite volume solver to establish which variations fall within the same order of accuracy as the baseline mesh.

\section{Mesh Generation Guideline Cases}

The meshing guidelines ${ }^{2}$ prescribed parameters that all mesh types needed to meet. These parameters were dependent on what resolution level was being constructed in a mesh family. The majority of these were accepted by participants and were nonissues. However, four guidelines were questioned by participants as being difficult to comply to, time-consuming, or they generated meshes of undesirable quality:

- The chordwise spacing at the leading and trailing edges were set to be approximately $0.1 \%$ of the local device chord measured for each element (slat, wing, and flaps).

- Spanwise spacing at the root and tips were requested to be $0.1 \%$ of the semispan.

- The number of points across the trailing edge was set based on the mesh level being generated. The coarse, medium, fine, and extra-fine mesh levels were expected to have $5,9,13$, and 17 points across the trailing edges respectively.

- Volume meshes were recommended to have at least 2 layers of constant cell spacing normal to the viscous walls and to use scaled stretching ratios in the boundary layer region across mesh family levels without exceeding a value of 1.25 .

The committee-supplied unstructured and hybrid meshes for the GMGW1, generated with Pointwise ${ }^{\circledR}$, complied with the guidelines with the exception of 2 layers of constant cell spacing normal to the viscous walls. This lack of compliance was due to a lack of functionality within Pointwise ${ }^{\circledR}$ to generate those constant layers in the mesh at the time. In the time since, that capability has been added to the version of Pointwise ${ }^{\circledR}$ used for this study.

Like the workshop participants, Woeber et al. ${ }^{3}$ noted that there were also conflicts in the guidelines that either made the mesh generation process take longer or created poor quality meshes. Mesh generation practitioners in both the participant and committee groups agreed that further work should be performed to determine which guidelines were necessary and which could be eliminated from the general set of recommended best practices for the community.

The baseline GMGW1 all tetrahedral medium-resolution mesh was the only mesh in the committee-supplied mesh family modified for this study. Since only that mesh was used, all mesh cases generated had the same initial cell height of 0.00117 off the viscous walls. This value was calculated based on a $y+$ of $2 / 3, R_{e}$ of 3.26 million, and characteristic reference length of 275.8 inches for the HL-CRM. Additionally, all cases started at the same initial growth rate of $1.16(16 \%)$ from the end of the constant cell layer region. The four cases devised to test the specific guidelines identified previously are defined in Table 1 based on the variations applied. They are described in detail in the following sections.

\section{A. Spanwise and Chordwise Spacing Modifications}

When possible, the meshing guideline variation listed for each case was the only modification applied to the baseline mesh. The one exception, which was unavoidable, was for Mod 1. When only the chordwise and spanwise spacings in the baseline mesh were adjusted, the aspect ratio in the spanwise direction on the slat, wing, and flap elements increased from a previous maximum value of 194 to 280. This led to a volume mesh of unacceptable quality in which the maximum included dihedral angle was 180 degrees. To improve the quality of the volume mesh, the spanwise number of mesh points on the leading and trailing edges of the slat, wing, and flaps was increased with the goal of getting the aspect ratio down to a value close to or lower than the one originally seen in the baseline mesh. To maintain the remaining original characteristics of the baseline mesh, this process had to be completed manually, which made it difficult to precisely reach the previous value for the aspect ratio. Since reducing the aspect ratio to 194 exactly would be an exercise of diminishing returns, 
Table 1. Mesh Cases by Guideline Variance.

\begin{tabular}{|c|c|c|c|c|}
\hline Case & Guideline Variance & $\begin{array}{c}\text { Total Mesh Size } \\
\text { (Points) }\end{array}$ & $\begin{array}{c}\text { Total Mesh Size } \\
\text { (Cells) }\end{array}$ & $\begin{array}{c}\text { Growth Rate } \\
\text { Acceleration }\end{array}$ \\
\hline Baseline & $\mathrm{N} \backslash \mathrm{A}$ & $26,491,087$ & $157,171,373$ & 1.0 \\
\hline Mod 1 & $\begin{array}{c}\text { Chordwise/Spanwise spacings } \\
\text { terminate at isotropy }\end{array}$ & $35,821,229$ & $212,499,662$ & 1.0 \\
\hline Mod 2a & $\begin{array}{c}\text { Constant boundary layer growth rate } \\
\text { 1 initial constant height cell layer }\end{array}$ & $27,141,622$ & $161,023,553$ & 1.0 \\
\hline Mod 2b & $\begin{array}{c}\text { Constant boundary layer growth rate } \\
2 \text { initial constant height cell layers }\end{array}$ & $27,710,515$ & $164,431,027$ & 1.0 \\
\hline Mod 3a & $\begin{array}{c}\text { Variable boundary layer growth rate } \\
1 \text { initial constant height cell layer }\end{array}$ & $16,393,975$ & $96,613,420$ & 1.0115 \\
\hline Mod 3b & $\begin{array}{c}\text { Variable boundary layer growth rate } \\
2 \text { initial constant height cell layers }\end{array}$ & $16,963,259$ & $100,024,105$ & 1.0115 \\
\hline Mod 4a & 7 points across trailing edge & $25,890,443$ & $153,625,292$ & 1.0 \\
\hline Mod 4b & 5 points across trailing edge & $24,983,799$ & $148,269,607$ & 1.0 \\
\hline Mod 4c & 3 points across trailing edge & $23,514,021$ & $139,576,394$ & 1.0 \\
\hline
\end{tabular}

it was decided that it would be best to instead refine each of these elements in a consistent manner (by the same factor of 2) in the spanwise direction. This resulted in a final surface mesh aspect ratio of 125 which, in turn, generated a tetrahedral volume mesh with a maximum maximum included dihedral angle of 177.4. This metric calculation for the Mod 1 mesh case was $0.42 \%$ less than the same calculated metric for the original baseline mesh.

Comparisons of the baseline and Mod 1 surface meshes at key trailing edge locations show the effects of these spacing changes on surface cell aspect ratio and area ratio metrics, see Figures 1, 2, and 3. Figure 1 compares the upper surface mesh in the vicinity of the root of the inboard flap, to include the trailing edge. If all mesh point spacings in this location are set equal to the minimum required spacing (i.e., the spacing derived from the required number of points across the trailing edge), a smoother mesh with higher quality, isotropic surface cells near the root end of the flap results (Figure 1). The net effect is to reduce anisotropy near the element ends. Similar results can be seen at the inboard/outboard flap trailing edges in the gap region (Figure 2) and at the wing tip (Figure 3).

In Figure 1, Figure 2, and Figure 3, it may be apparent to the reader that in some cases it appears there are surface cells with high aspect or area ratios in the Mod 1 mesh. In all of these cases, even though these cells exceeded what is generally recommended for ideal aspect and area ratios, their maximum value is still significantly less than those for the baseline mesh. Further mesh modifications, such as local edge refinement, would resolve/improve the quality of these cells. However, since the focus of this study was to study individual variations on the baseline mesh, additional modifications were avoided whenever possible so that the effect of the individual variation (such as a spacing change) could be seen clearly.

\section{B. Constant Cell Height Layers Modifications with Constant Geometric Growth Rate}

Meshes generated for Mod $2 a$ and Mod $2 b$ focused on modifying the baseline volume mesh to use 1 or 2 layers of cells respectively with a height of 0.00117 off the viscous walls, followed by successive cell layer heights growing at a constant geometric growth rate of $1.16(16 \%)$ through the remainder of the boundary layer region. The resulting viscous meshing region off the wall contained 60 layers at maximum viscous layer region heights of approximately 34.5 and 20.2 inches over the wing and flap, respectively, for Mod $2 a$ and $\operatorname{Mod} 2 b$ based on a cut through the mesh at $Y=638(\eta=0.552)$. This location can be seen in Figure 4 and will be used as a reference in this and the following sections.

An overall view of the viscous region over all elements is shown in Figure 5(a) with a detail of the region over the flap shown in Figure 5(b) for the Mod $2 b$ mesh. Only the Mod $2 b$ mesh is shown since the mesh profile is very similar between the two meshes at this zoom-level.

Inspection of the initial surface layers intersecting the symmetry plane (Figure 6) and at locations along the slat, wing, and flaps verified that the correct number of constant cell height layers were being generated. 


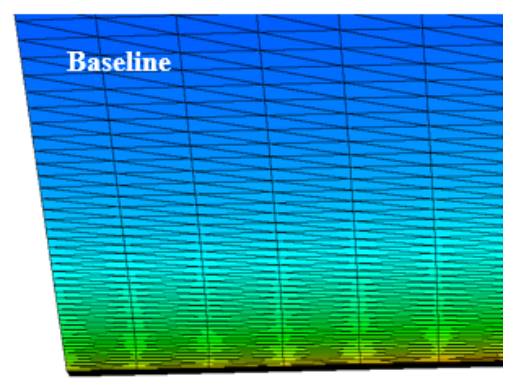

(a) Baseline Aspect Ratio

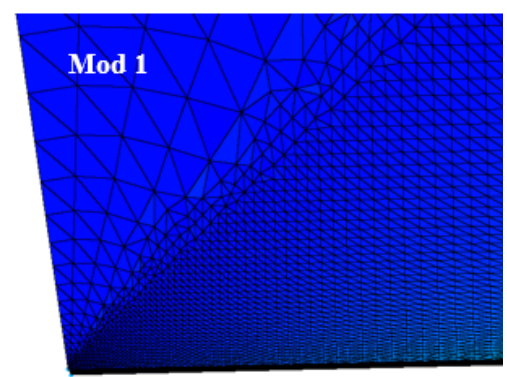

(c) Mod 1 Aspect Ratio

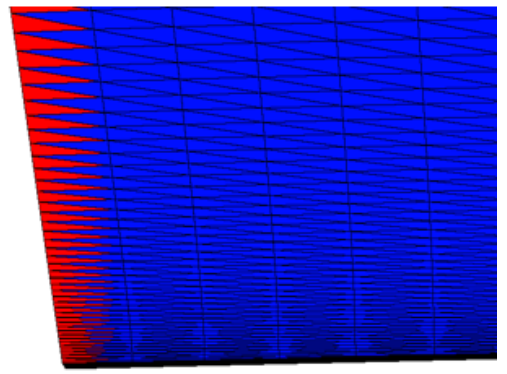

(b) Baseline Area Ratio

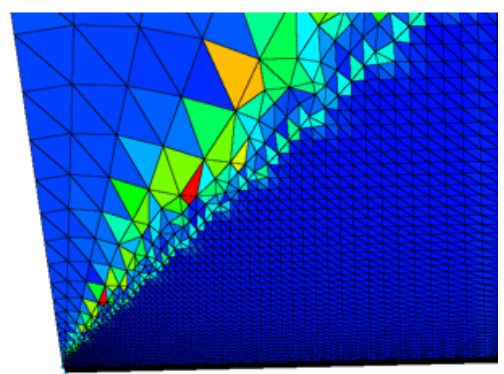

(d) Mod 1 Area Ratio
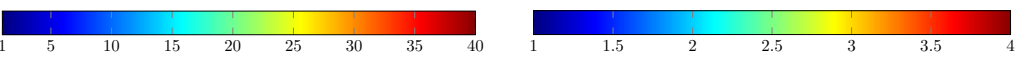

Figure 1. Comparisons of surface mesh cell aspect ratio and area ratio of the root end of the inboard flap.

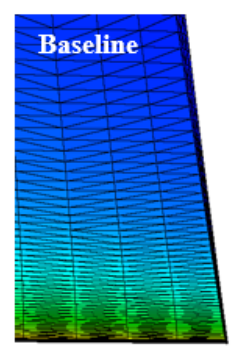

(a) Baseline Aspect Ratio
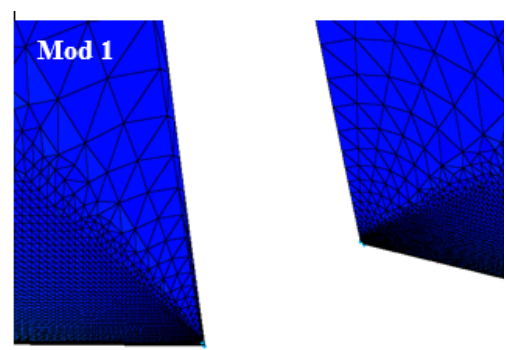

(c) Mod 1 Aspect Ratio
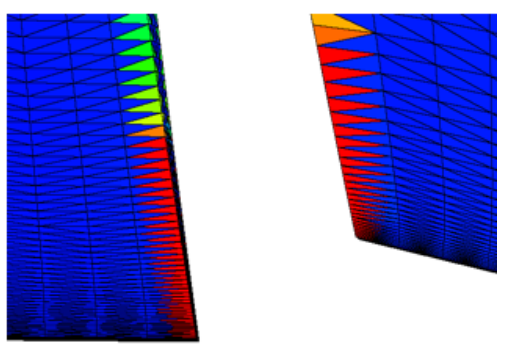

(b) Baseline Area Ratio
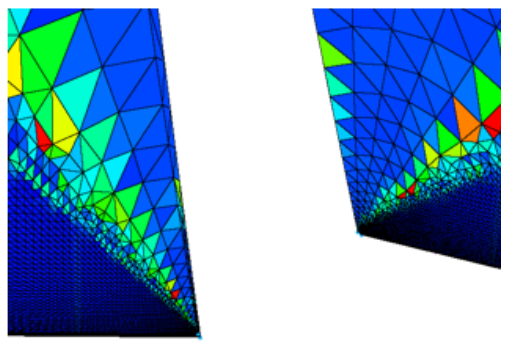

(d) Mod 1 Area Ratio
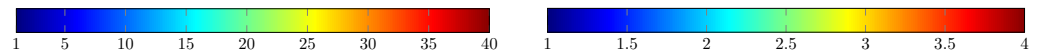

Figure 2. Comparisons of surface mesh cell aspect ratio and area ratio of the inboard and outboard flaps at the flap gap. 


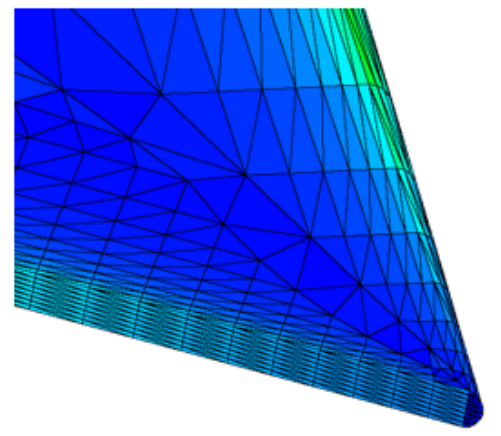

(a) Baseline Aspect Ratio

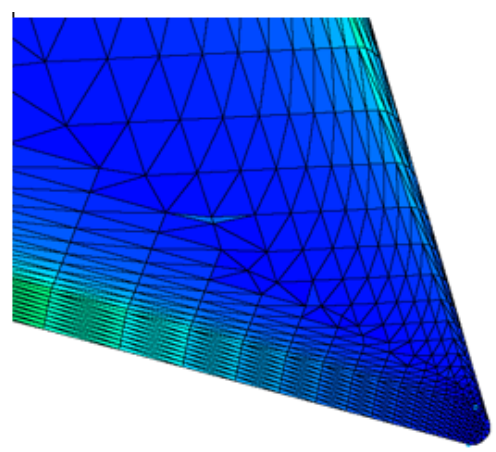

(c) Mod 1 Aspect Ratio

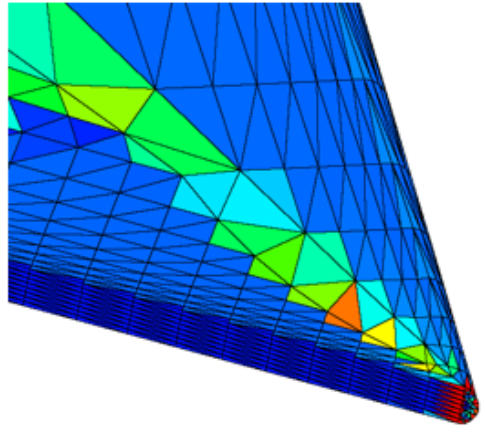

(b) Baseline Area Ratio

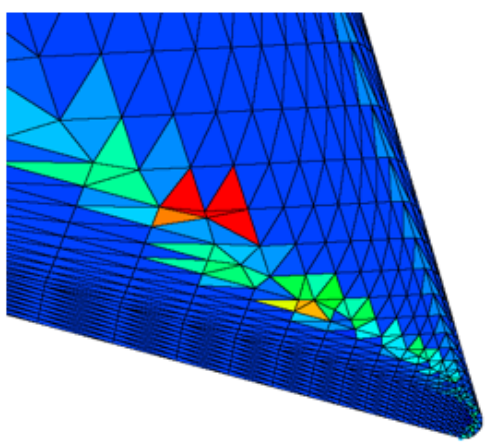

(d) Mod 1 Area Ratio

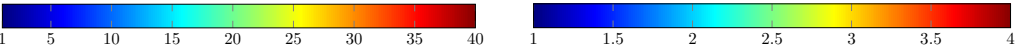

Figure 3. Comparisons of surface mesh cell aspect ratio and area ratio of the wing tip.

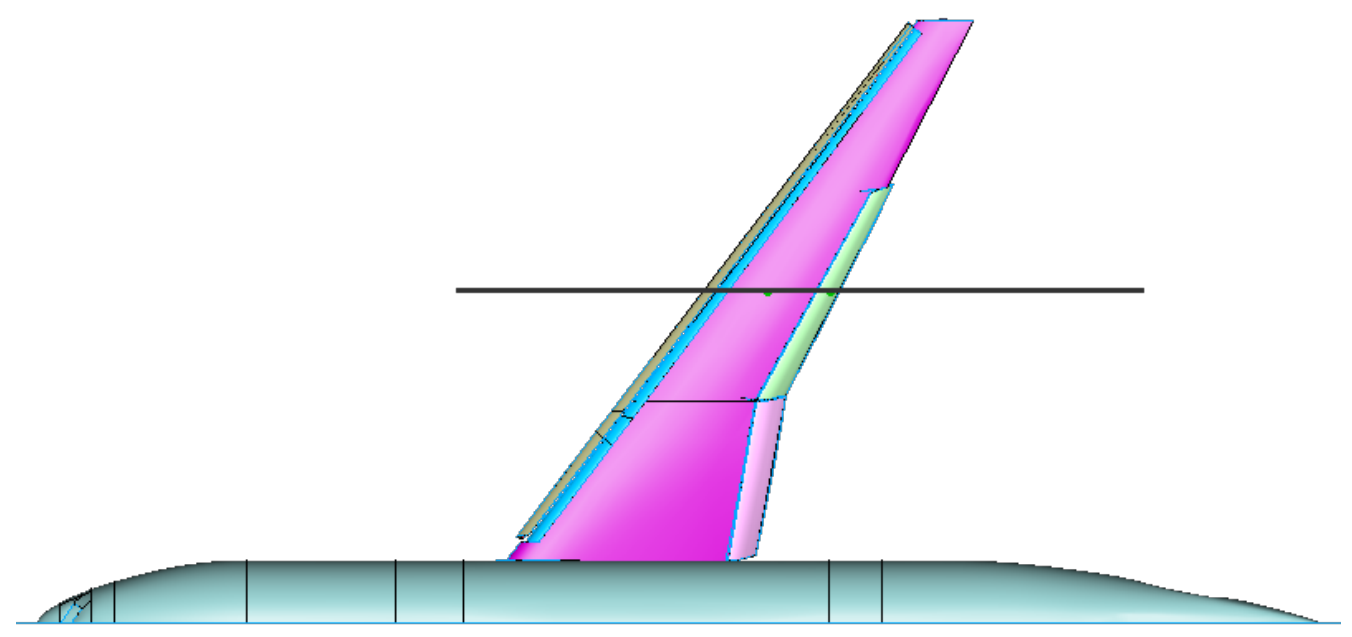

Figure 4. A cut location of $Y=638(\eta=0.522)$ used for all referenced mesh figures. 


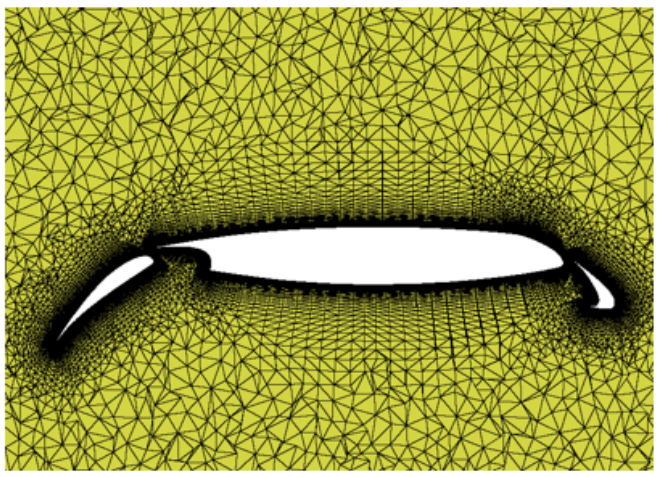

(a) Slat, main, and flap

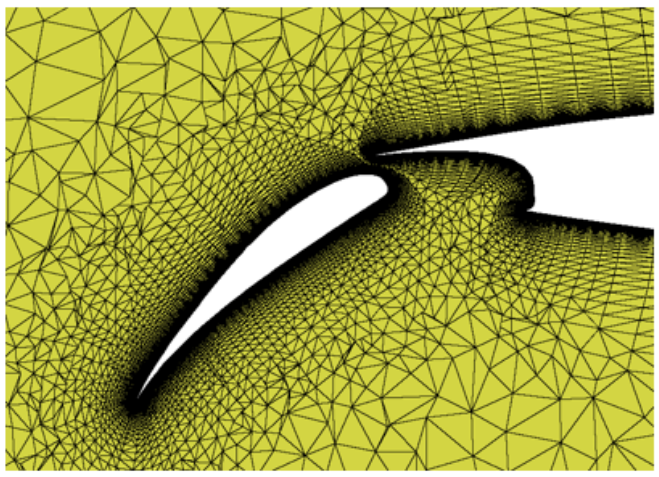

(b) Flap detail

Figure 5. A $Y=638$ cut shows the boundary layer mesh for the Mod $2 b$ mesh.

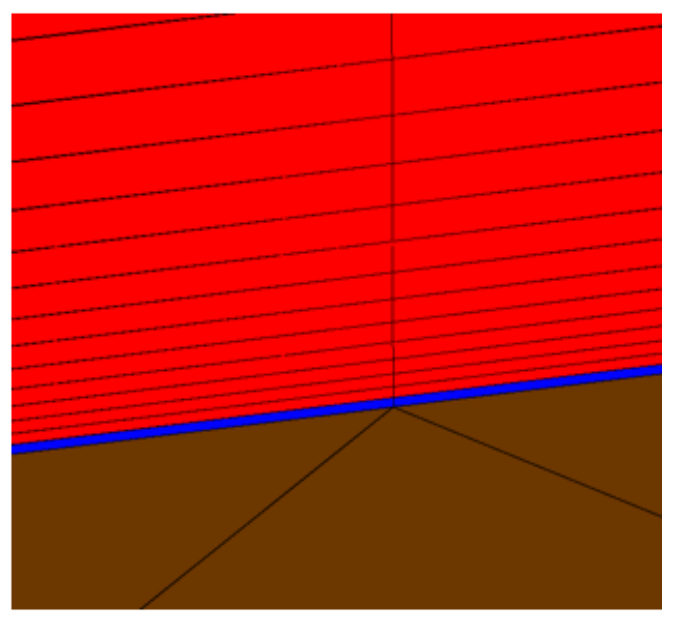

(a) $\operatorname{Mod} 2 a$

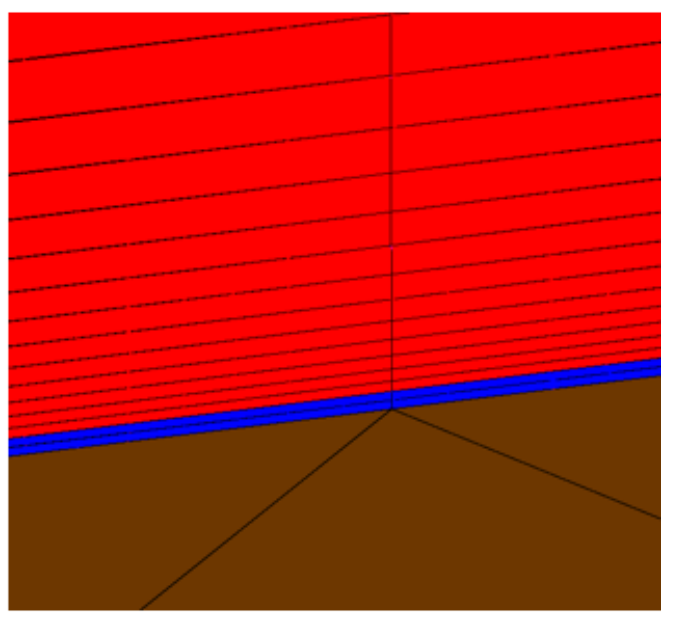

(b) $\operatorname{Mod} 2 b$

Figure 6. Comparison of Mod $2 a$ and Mod $2 b$ meshes on the $\mathrm{Y}$ symmetry plane with 1 and 2 layers of constant cell heights (in blue) off the viscous walls (in brown). 


\section{Constant Cell Height Layers Modifications with Variable Growth Rate}

Discussions were noted amongst participants and some committee members on whether guidelines should address using a constant or a variable growth rate within the viscous boundary layer region of the mesh during the $1^{\text {st }}$ and $2^{\text {nd }}$ HLPWs. Additional discussion on this topic also arose in at least one of the breakout sessions from GMGW1. This interest warranted the development of the Mod $3 a$ and Mod $3 b$ meshes.

Meshes generated for Mod $3 a$ and Mod $3 b$ focused on modifying the baseline volume mesh to use 1 or 2 layers of cells with a height of 0.00117 off the viscous walls, respectively, followed by successive cell layer heights starting at an initial growth rate of $1.16(16 \%)$. A growth rate acceleration of $1.0115(1.15 \%)$ was then applied to each layers growth rate. Since the initial cell height and growth rate were fixed from the meshing guidelines, a law of the wall computation for a flat plate with the HLPW3 flow parameters was used to determine an appropriate growth rate acceleration. Note that the flat plate calculation predicted initial cell heights and growth rates that were close but not identical to those prescribed by the meshing guidelines. The resulting viscous meshing region off the wall contained 30 layers at maximum viscous layer region heights of approximately 14.8 and 7.1 over the wing and flap, respectively, for $\operatorname{Mod} 3 a$ and $\operatorname{Mod} 3 b$ at a cut through the mesh at $Y=638(\eta=0.552)$. Note that the number of layers and heights are roughly half of the Mod 2 cases due to the earlier termination of the boundary layer structures as cell heights reached the isotropic freestream values closer to the surface due to the growth rate acceleration.

An overall view of the viscous region over all elements is shown in Figure 7(a) with a detail of the region over the flap shown in Figure 7(b) for the Mod $3 b$ mesh. Only the Mod $3 b$ mesh is shown since the mesh profile is very similar between the two meshes at this zoom-level.

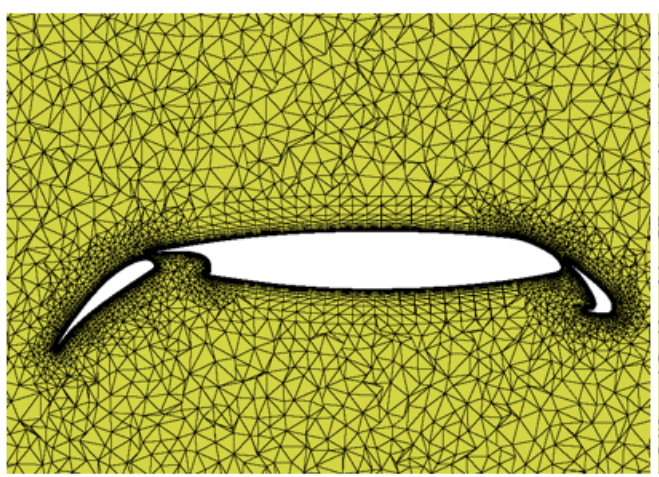

(a) Slat, main, and flap

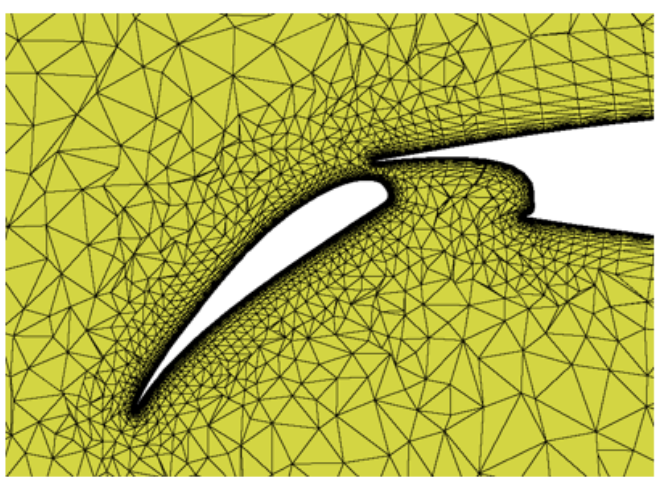

(b) Flap detail

Figure 7. A $Y=638$ cut shows the boundary layer mesh for the Mod $3 b$ mesh.

Inspection of the initial layers off the surface on the symmetry plane and at locations along the slat, wing, and flaps verified that the correct number of constant cell height layers were being generated in both meshes. Additionally, comparisons were made in the boundary layer mesh profiles between the Mod $2 a / 2 b$ and the Mod 3a/3b meshes (Figure 8). Again, the growth rate acceleration applied to the Mod $3 a$ and Mod $3 b$ meshes can be seen roughly cut in half the total height of the viscous cell layers in the mesh (Figures 5 and 7 ).

\section{Trailing Edge Point Resolution Modifications}

The final set of modifications involved varying the number of cells or points across the trailing edge (TE) of each element. For the medium resolution baseline mesh, 9 points ( 8 cells) were required across the TE in the meshing guidelines. In $\operatorname{Mod} 4 a, \operatorname{Mod} 4 b$, and $\operatorname{Mod} 4 c$, the number of points across the trailing edges was reduced to 7,5 , and 3 , respectively. The upper and lower element surface meshes were designed to maintain the initial cell height around the corner of the trailing edge (in the chordwise-direction) via an adjacent mesh T-Rex ${ }^{6}$ boundary condition. As the number of points across the trailing edge was modified for this case, the upper and lower surface meshes were updated accordingly so that anisotropic triangular surface cells marched off the trailing edge by the same mesh point spacing. This effect was relatively local to the trailing edge and was considered an important mesh feature to retain so as to keep mesh area ratio reasonable in this region. A comparison of the Mod $4 a, \operatorname{Mod} 4 b$, and $\operatorname{Mod} 4 c$ meshes at a $\mathrm{Y}=638$ cut and focused on the wing and flap trailing edges shows how the resolution of the mesh off the trailing edge was successively coarsened (Figure 9). 


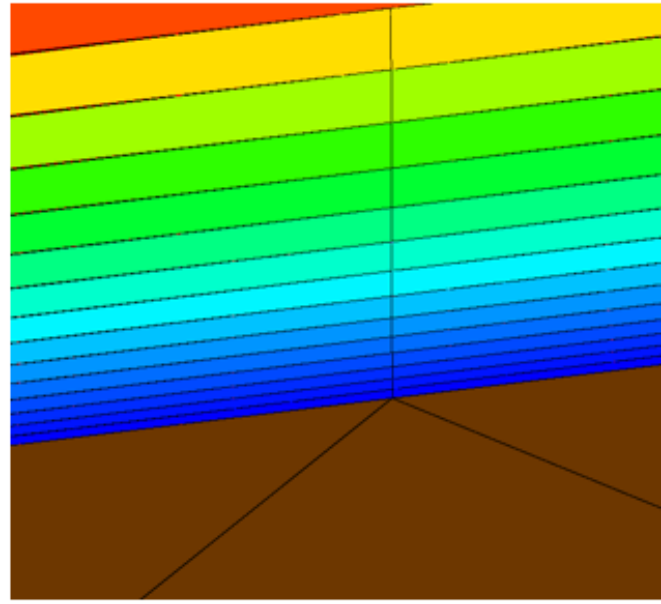

(a) Mod $2 a$

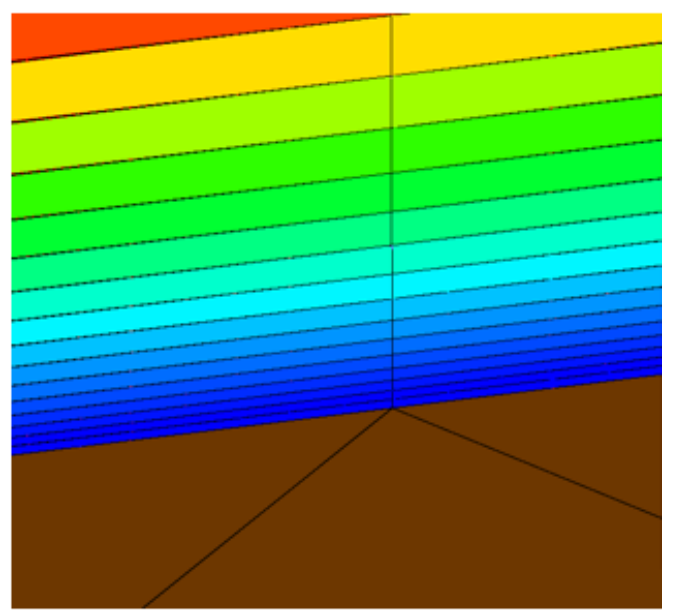

(c) $\operatorname{Mod} 2 b$

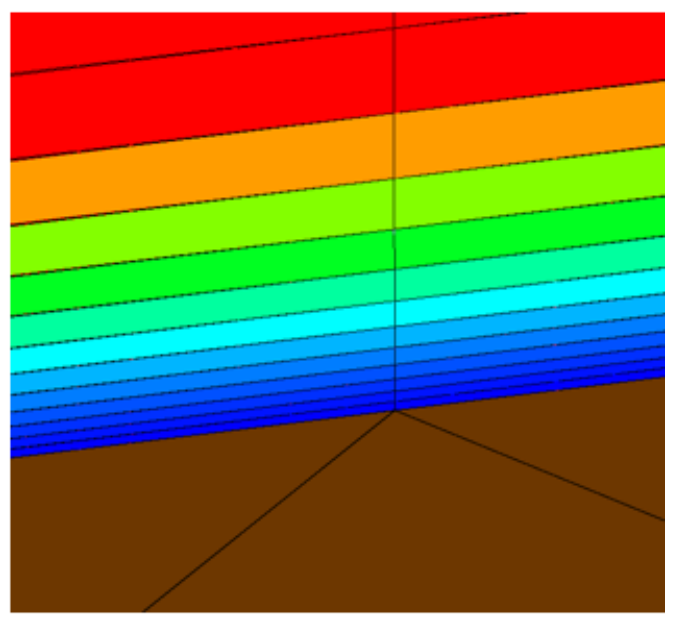

(b) Mod $3 a$

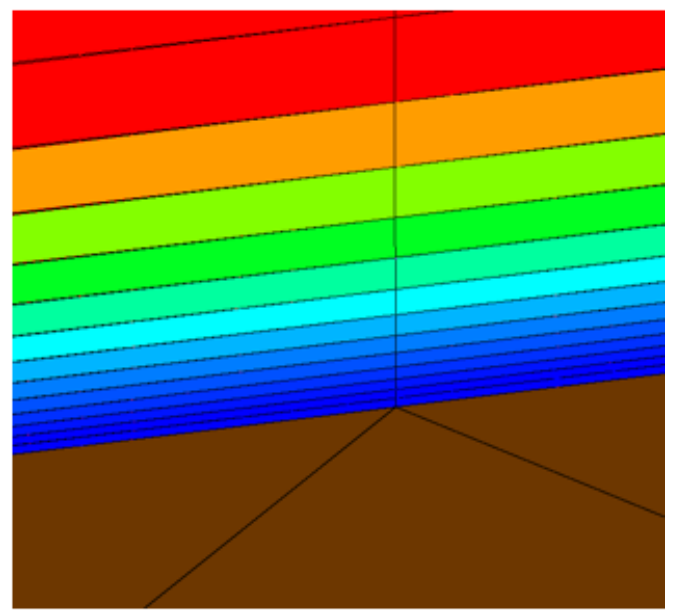

(d) $\operatorname{Mod} 3 b$

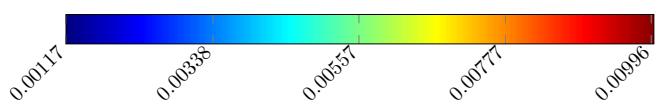

Figure 8. Comparison of boundary layer growth between the $\operatorname{Mod} 2 a, \operatorname{Mod} 2 b, \operatorname{Mod} 3 a$, and $\operatorname{Mod} 3 b$ meshes on the Y symmetry plane normal to the viscous walls (in brown). Contours represent normal edge length. 


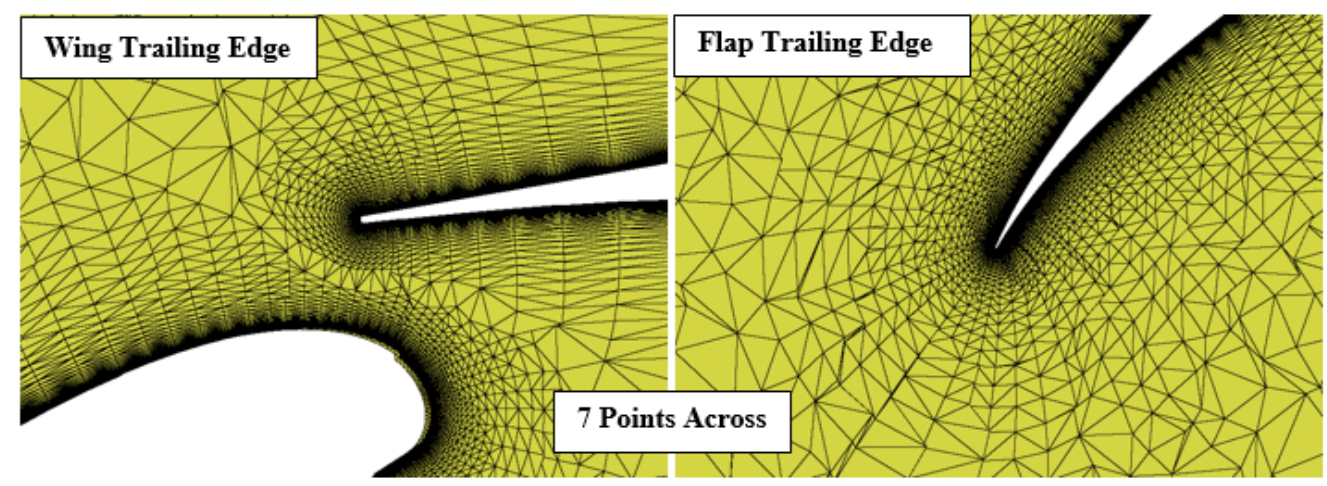

(a) $\operatorname{Mod} 4 a$

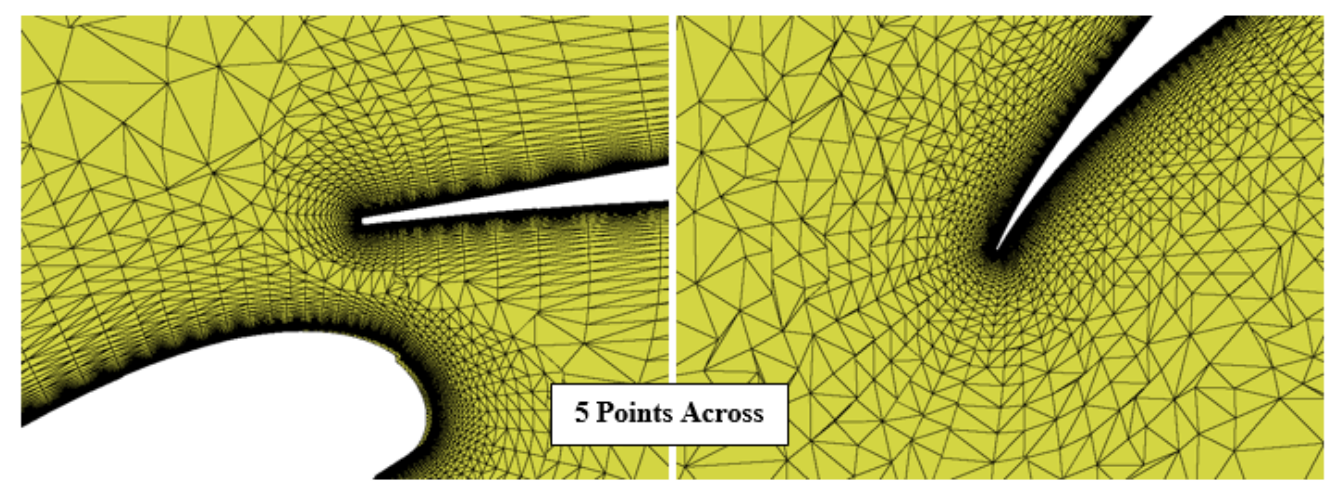

(b) $\operatorname{Mod} 4 b$

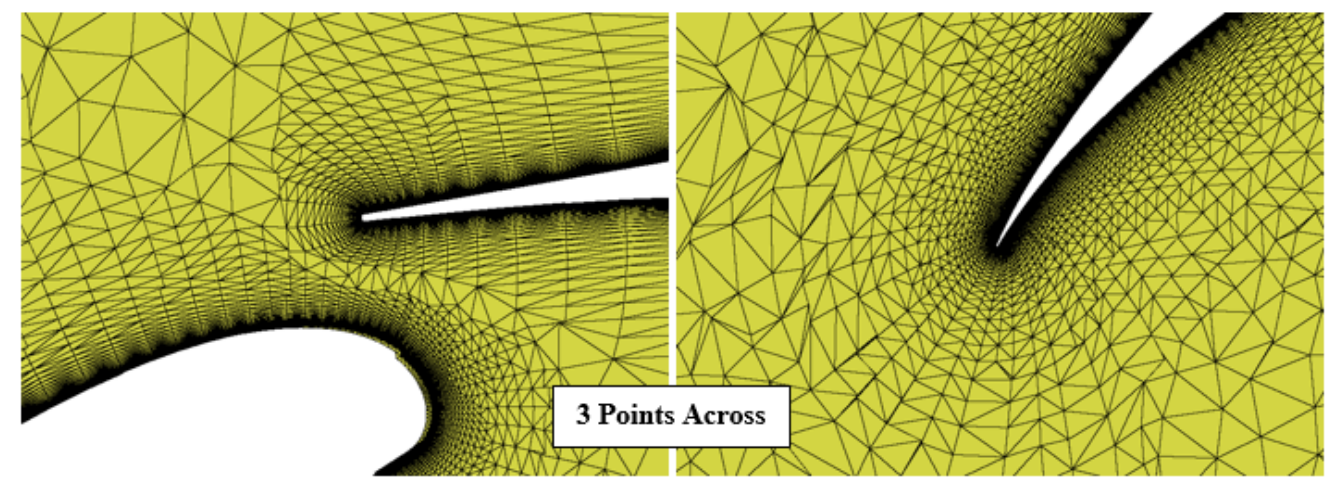

(c) $\operatorname{Mod} 4 \mathrm{c}$

Figure 9. Effect of varying the number of points across the blunt Trailing Edges represented by a cut through the Mod $4 a$, Mod $4 b$, and Mod $4 c$ meshes at $Y=638$ inches. 


\section{E. Mesh Case Size and A Priori Metrics}

As can be expected, some of these modifications made a significant difference in overall mesh size when compared to the baseline mesh from which they were derived. Figure 10 and Table 2 illustrate how much each of the mesh modifications varied from the total mesh size of the baseline. Mod 1, Mod 3a, and Mod $3 b$ cases saw the largest deviations in mesh size at $+26 \%,-61 \%$, and $-55 \%$ from the baseline, respectively.

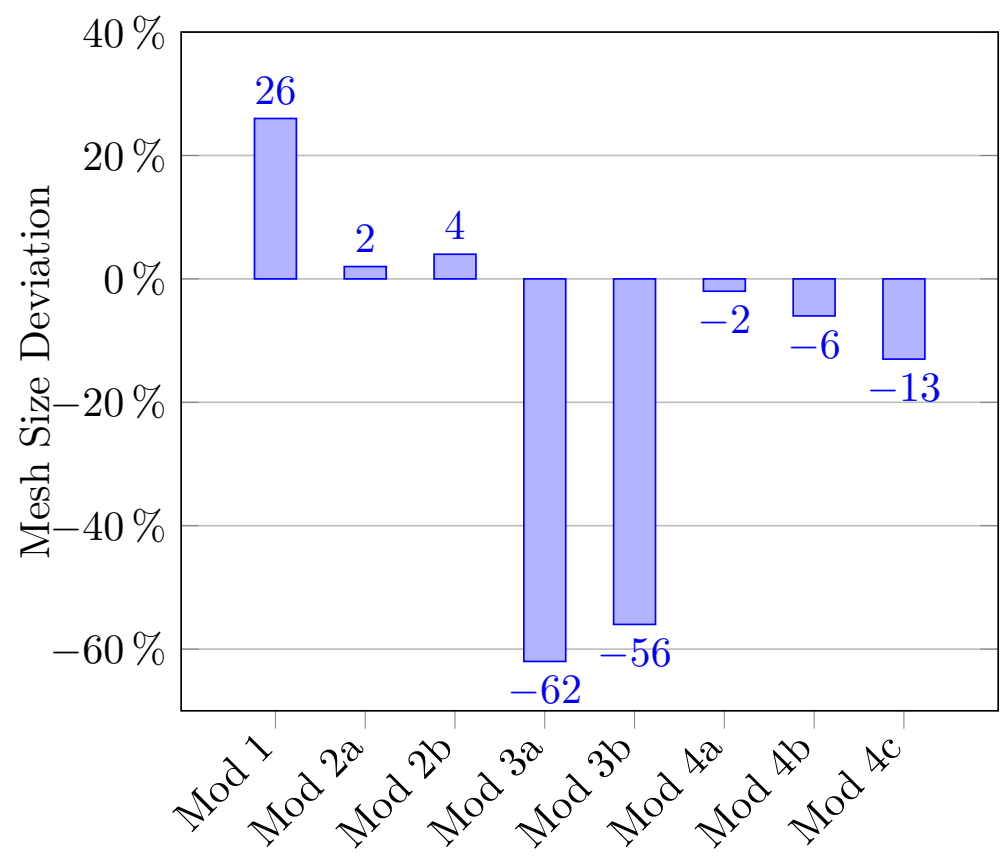

Figure 10. Mesh size deviation due to guideline variance.

Table 2. Mesh Quality Metric Comparisons.

\begin{tabular}{|c|c|c|c|}
\hline \multirow{2}{*}{} & \multicolumn{3}{|c|}{ Max. Included Angle } \\
\cline { 2 - 4 } & Min & Avg & Max \\
\hline Baseline & 71.3 & 95.0 & 178.1 \\
\hline Mod 1 & 70.9 & 94.9 & 177.4 \\
\hline Mod 2a & 70.9 & 95.1 & 178.9 \\
\hline Mod 2b & 70.9 & 95.1 & 177.6 \\
\hline Mod 3a & 71.3 & 95.1 & 177.8 \\
\hline Mod 3b & 71.3 & 94.9 & 177.9 \\
\hline Mod 4a & 71.3 & 95.1 & 177.9 \\
\hline Mod 4b & 71.2 & 95.1 & 178.1 \\
\hline Mod 4c & 71.1 & 95.4 & 178.8 \\
\hline
\end{tabular}

Examining the a priori metrics related to the mesh modifications revealed an important lesson that was reinforced in this study. Changes to control mesh parameters do not happen in a vacuum. Each modification made in a mesh, no matter how small, can have a waterfall effect that can necessitate other modifications. Improving the quality of a surface or volume mesh in one area can result in the quality worsening in another. If one is aware well ahead of time that you will be subsequently modifying a mesh, the topology can be designed in such a way as to mitigate this waterfall effect. This is commonly employed when there is an expectation that components in the geometry, and thus the mesh, will be swapped out for new designs later on. The mesh generation practitioner, armed with that knowledge, can use their expertise to plan a topology that will facilitate geometry/mesh component swapping to isolate these modifications, minimizing the effect on the remainder of the mesh. In GMGW1, the baseline mesh was not created with this intent so modifications 
made to the mesh to vary guidelines in this study were seen to only improve the quality of the mesh locally for that area, not necessarily for the mesh as a whole. As an example, a review of the maximum included angle for tetrahedra given in Table 2 shows that the deviations in the worst quality cell value are minor, i.e., $<1 \%$. The best approach to achieve a significant improvement in quality in the entire mesh would be to use a combination of the guideline modifications that provide a reduction in time and effort while maintaining solution convergence and accuracy.

\section{Flow Solution}

For the evaluation of the mesh guideline variances, we utilize the FUN3D Computational Fluid Dynamics solver developed at the NASA Langley Research Center. FUN3D is an unstructured, node-centered, secondorder, finite-volume flow solver initiated by Anderson and Bonhaus. ${ }^{7,8}$ The software is continually developed by a team of researchers at NASA Langley and provides a number of capabilities to incorporate simulation of complex, moving body dynamics, multiphysics simulation, and adjoint-based design. ${ }^{9}$ The discrete form of the governing equations are solved either in a time-accurate manner with a constant time step at every mesh point or with variable time stepping to accelerate convergence to a steady state. At each iteration step, a linear system of equations is relaxed with a point implicit procedure. ${ }^{10}$ FUN3D can accommodate multiple mesh element types including tetrahedra, prism, pyramid and hexahedra. For the current work, Roe's fluxdifference splitting scheme ${ }^{11}$ is used without a flux limiter. Although several turbulence models are available in FUN3D, ranging from the one-equation Spalart-Allmaras (SA) ${ }^{12}$ model to full Reynolds Stress models, the current solutions were obtained using the SA model with rotation/curvature correction (i.e., SA-RC). All meshes were comprised of only tetrahedral elements. The conditions were taken from the HLPW3 "Case 1: Grid Convergence Study", namely: $M=0.2 ; \mathbf{R}_{\mathbf{e}}=3.26 x 10^{6} ; \alpha=8^{\circ}$ and $16^{\circ}$.

The present solutions were obtained on an SGI ICE Altix X computing cluster utilizing 25 dual socket 8 core 2.60 GHz Intel E5-2670 Sandybridge processor nodes for a total of 400 cores. Each node was equipped with 32GB of RAM. The FUN3D code was run in a steady mode for all meshes including the HLPW3 baseline and those resulting from the guideline variances for a total of 10,000 iterations on each mesh requiring less than 6 hours per simulation. For all cases, the standard deviation of $\mathrm{C}_{\mathrm{L}}$ over the final 1000 iterations was $\mathcal{O}\left(10^{-5}\right)$

\section{Results}

Details of the convergence history for the baseline mesh and the 8 meshing guideline variances at 8 and 16 degrees angle of attack are provided in Figure 11. Plotted are the density and turbulence model residuals along with the lift coefficient. It can be seen that all of the variances maintain very good convergence histories with most closely following that of the baseline mesh. Cases Mod $3 a$ and Mod $3 b$ show slightly faster convergence for both density and the turbulence model while still maintaining the general trend of the baseline case. At $\alpha=16^{\circ}$, cases $\operatorname{Mod} 2 a$ and $\operatorname{Mod} 2 b$ exhibit some minor instability in the density and turbulence model residuals after 8000 steps, however, there is little, if any, impact on $\mathrm{C}_{\mathrm{L}}$.

Again, the solutions were deemed to have been sufficiently converged for the purposes of this work in that the values of $\mathrm{C}_{\mathrm{L}}$ showed little variation over the final 1000 iteration steps, having a standard deviation over that interval $\mathcal{O}\left(10^{-5}\right)$. This data has been tabulated for all cases at both angles of attack in Tables 3 and 4 . From this data, it is observed that the meshing guideline variance with the most significant impact on $\mathrm{C}_{\mathrm{L}}$ was that of $\operatorname{Mod} 1$. However, this guideline variance only impacted the final $\mathrm{C}_{\mathrm{L}}$ by $-3.33 \%$ at $\alpha=8^{\circ}$ and $1.69 \%$ at $\alpha=16^{\circ}$. The next largest impact of guideline variance on $\mathrm{C}_{\mathrm{L}}$ occurs for cases Mod $4 \mathrm{~b}$ and $\operatorname{Mod} 4 \mathrm{c}$ at $\alpha=8^{\circ}$. Here the percent error from the baseline solution was $-1.40 \%$ and $-1.56 \%$, respectively. All other meshing guideline variances produced less than $1.0 \%$ error with respect to that of the baseline mesh in the final $\mathrm{C}_{\mathrm{L}}$.

In order to better assess the impact of the meshing guideline variances on the solution, streamwise velocity profiles were extracted from the solution at the two locations shown in Figure 12. Both locations are at $Y=638$ inches spanwise. The first location, at $X=1495$ inches longitudinally, is on the main element at roughly midchord. The second location, at $X=1615$ inches longitudinally, is on the outboard flap. These locations are consistent with locations used by the HLPW3 committee to assess participant solutions. ${ }^{13}$

The extracted, normalized, streamwise velocity profiles at $Y=638$ inches and $X=1495$ and $X=1615$ inches are shown in Figure 13 for the $\alpha=8^{\circ}$ condition. The influence of the slat wake is observed as slight 


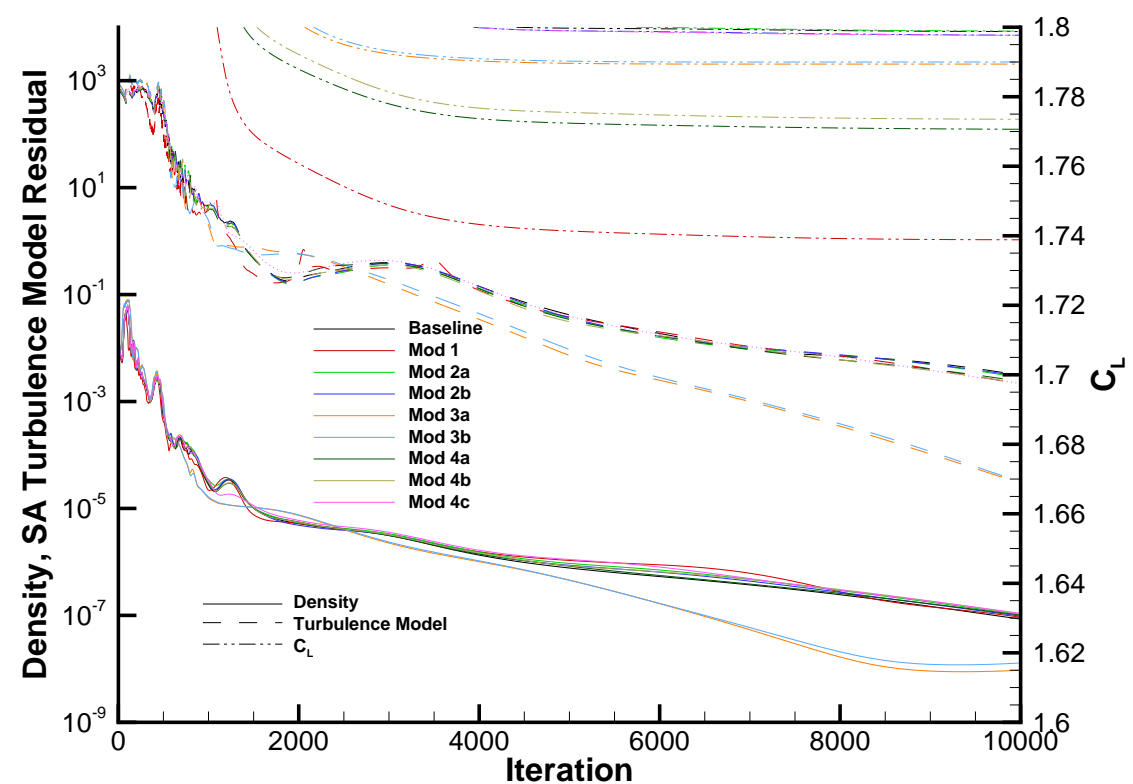

(a) Convergence history at $\alpha=8^{\circ}$

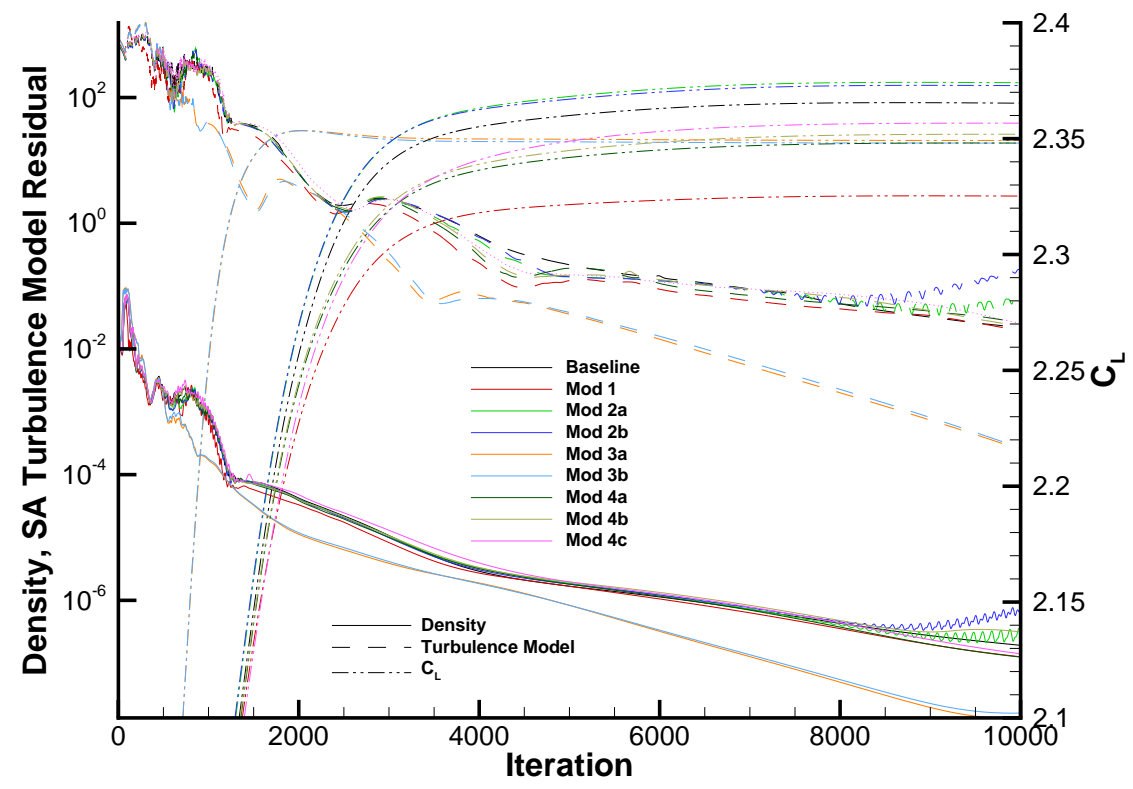

(b) Convergence history at $\alpha=16^{\circ}$

Figure 11. Convergence history: Density and SA Turbulence Model Residuals, Lift Coefficient. 
Table 3. $\mathbf{C}_{\mathrm{L}}$ at $\alpha=8^{\circ}$.

\begin{tabular}{|c|c|c|c|c|}
\hline Case & $\mathrm{C}_{\mathrm{L}}$ & \% Error & $\left.\begin{array}{c}\text { Mean } \\
\text { Final } \\
1000 \\
\text { steps }\end{array}\right)$ & $\begin{array}{c}\text { Standard } \\
\text { Deviation } \\
\left(\begin{array}{c}\text { Final } \\
1000 \\
\text { steps }\end{array}\right)\end{array}$ \\
\hline Baseline & 1.7987 & 0.00 & 1.7988 & $2.79 \mathrm{e}-5$ \\
\hline Mod 1 & 1.7389 & -3.33 & 1.7389 & $2.64 \mathrm{e}-5$ \\
\hline Mod 2a & 1.7989 & 0.01 & 1.7989 & $3.57 \mathrm{e}-5$ \\
\hline Mod 2b & 1.7977 & -0.06 & 1.7978 & $3.72 \mathrm{e}-5$ \\
\hline $\operatorname{Mod} 3 \mathrm{a}$ & 1.7894 & -0.52 & 1.7894 & $1.62 \mathrm{e}-7$ \\
\hline $\operatorname{Mod} 3 \mathrm{~b}$ & 1.7900 & -0.49 & 1.7900 & $3.17 \mathrm{e}-7$ \\
\hline $\operatorname{Mod} 4 \mathrm{a}$ & 1.7977 & -0.06 & 1.7977 & $3.32 \mathrm{e}-5$ \\
\hline $\operatorname{Mod} 4 \mathrm{~b}$ & 1.7735 & -1.40 & 1.7736 & $3.28 \mathrm{e}-5$ \\
\hline $\operatorname{Mod} 4 \mathrm{c}$ & 1.7706 & -1.56 & 1.7707 & $3.77 \mathrm{e}-5$ \\
\hline
\end{tabular}

Table 4. $\mathbf{C}_{\mathrm{L}}$ at $\alpha=16^{\circ}$.

\begin{tabular}{|c|c|c|c|c|}
\hline Case & $\mathrm{C}_{\mathrm{L}}$ & \% Error & $\left.\begin{array}{c}\text { Mean } \\
\text { Final } \\
1000 \\
\text { steps }\end{array}\right)$ & $\begin{array}{c}\text { Standard } \\
\text { Deviation } \\
\left(\begin{array}{c}\text { Final } \\
1000 \\
\text { steps }\end{array}\right.\end{array}$ \\
\hline Baseline & 2.3653 & 0.00 & 2.3654 & $6.20 \mathrm{e}-5$ \\
\hline Mod 1 & 2.3252 & -1.69 & 2.3253 & $3.03 \mathrm{e}-5$ \\
\hline Mod 2a & 2.3741 & 0.37 & 2.3742 & $4.45 \mathrm{e}-5$ \\
\hline Mod 2b & 2.3729 & 0.32 & 2.3730 & $3.79 \mathrm{e}-5$ \\
\hline Mod 3a & 2.3492 & -0.68 & 2.3492 & $4.73 \mathrm{e}-6$ \\
\hline Mod 3b & 2.3481 & -0.73 & 2.3481 & $5.73 \mathrm{e}-6$ \\
\hline Mod 4a & 2.3567 & -0.36 & 2.3567 & $1.00 \mathrm{e}-5$ \\
\hline Mod 4b & 2.3519 & -0.57 & 2.3519 & $1.47 \mathrm{e}-5$ \\
\hline Mod 4c & 2.3481 & -0.73 & 2.3481 & $7.27 \mathrm{e}-6$ \\
\hline
\end{tabular}

velocity deficit in Figure 13(a) at roughly $Z=240$ inches. The majority of the guideline variances show minimal impact on the velocity profile. However, the Mod $3 a$ and Mod $3 b$ cases fail to adequately capture the wakes of the upstream elements. At $\alpha=8^{\circ}$, the $\operatorname{Mod} 3$ cases show no indication of the slat wake velocity deficit observed in the other solutions. While there is some velocity deficit representing the upstream wakes on the flap in Figure 14(a), Mod 3 again significantly underpredicts the velocity deficit. This is likely due to the early termination of the boundary layer structures in the mesh due to the growth rate acceleration parameter. This parameter forces a faster increase in cell height as the distance from the surface increases. The layers therefore terminate earlier as they reach a cell height equal to the isotropic freestream value closer to the surface. The net result is a lower mesh resolution in the vicinity of the wakes. Refer again to Figures 5, 7, and 8 for the differences. Note that none of the meshes, including the baseline, have the resolution necessary to distinguish the slat wake from the main element wake over the flap.

The Mod $2 a$ and Mod $2 b$ cases essentially overplot themselves. This is not surprising in that the variance associated with Mod 2 is limited to the addition of layers with constant cell height immediately adjacent to the viscous surfaces. It is believed that this guideline was based on the experience of the HLPW committee with the NASA OVERFLOW ${ }^{14}$ CFD tool with respect to the calculation of skin friction. ${ }^{15}$ Using the OVERFLOW tool, mesh generation guidelines have been established for accurate calculation of drag. "When the flow solver uses first-order one-sided differencing for the [mesh] metrics at the wall, several points of constant spacing should be used." ${ }^{16}$ The current work did not concern itself with drag computation and, as such, skin friction was not considered in the assessment of meshing guidelines for high lift configurations.

Similarly, the $\operatorname{Mod} 4 a$ and $\operatorname{Mod} 4 b$ cases at $\alpha=8^{\circ}$ overplot. This would indicate that for this node-centered solver, 5 points across the blunt trailing edges is sufficient. There is, however, a distinguishable difference in the flap velocity profiles when only 3 points are used in $\operatorname{Mod} 4 c$, indicating perhaps this resolution is too coarse at the medium level of mesh fidelity.

The slight spread in the velocity profiles is not entirely surprising in that the mesh selected for this study was the GMGW1 medium mesh. Therefore, these solutions are not mesh independent and the guideline variances, albeit small and localized, do produce slightly modified meshes. The result is a slight spread in the data. At $\alpha=8^{\circ}$, the spread is quite small if one neglects the Mod 3 cases. The spread in the profiles is slightly larger at $\alpha=16^{\circ}$, however, the general character is represented by all cases except for Mod 3 .

The upstream element wakes are more pronounced at the $\alpha=16^{\circ}$ condition of Figure 14 where the higher angle of attack produces stronger velocity deficits. Again, at both profile locations, the Mod 3 cases underpredict the velocity deficit. A slightly larger spread is present in the velocity profiles off the flap element of Figure 14(b), however, all cases, except Mod 3, exhibit the same character of inflection as the baseline. For convenience, Figure 15 provides a detail of the velocity deficit resulting from the main element wake. From this detail, cases Mod $2 a$ and $\operatorname{Mod} 2 b$ best reproduce the baseline velocity profile, however, Mod 1, Mod 4a, and $\operatorname{Mod} 4 \mathrm{~b}$ are very tightly grouped and show fairly good agreement with the baseline. Cases Mod 3 and Mod $4 c$ show less favorable agreement, where $\operatorname{Mod} 3$ again underpredicts the velocity deficit. 


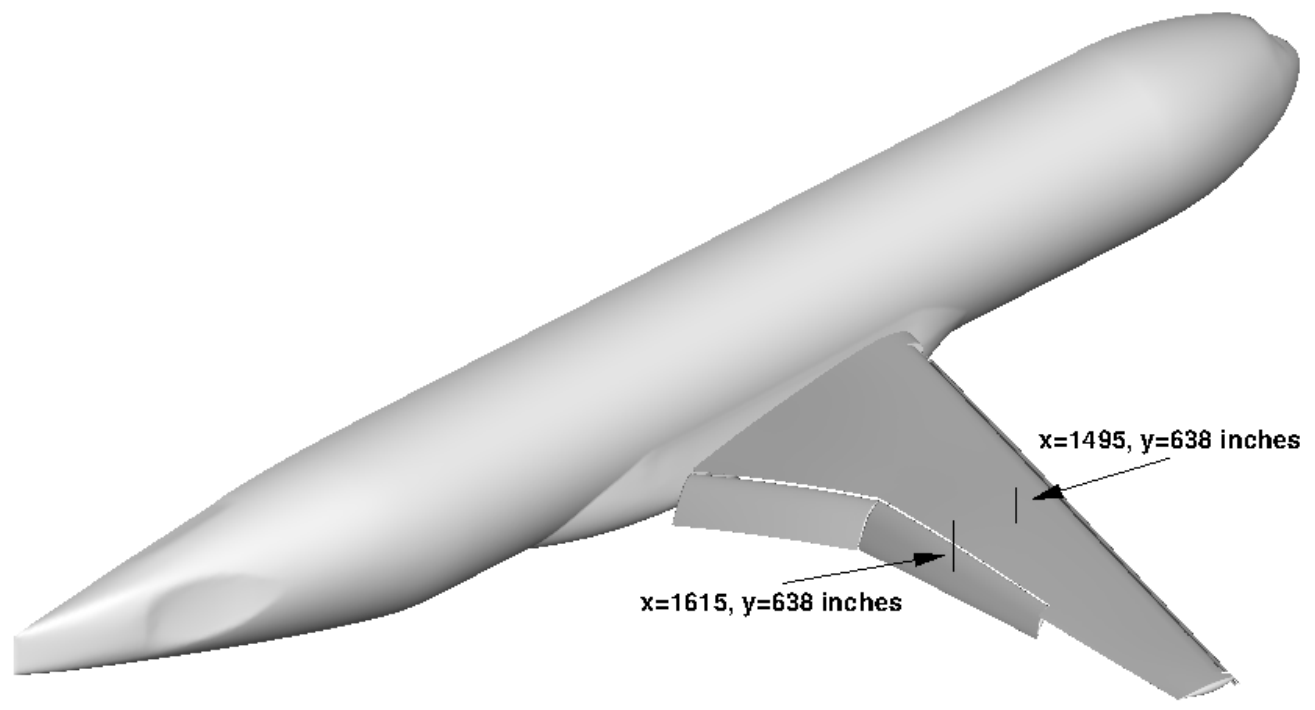

Figure 12. Location of velocity profiles.

\section{Conclusions}

A study was performed to apply variances to the guidelines imposed by the $3^{\text {rd }}$ High Lift Prediction Workshop on unstructured tetrahedral meshes generated to represent the High Lift Common Research Model geometry. The study targeted four guidelines based on questions raised by GMGW1 and HLPW3 participants. Variances of varying degree were applied to the targeted guidelines. The variances were applied individually to independently assess their impact on the resulting mesh and computed flow solution. Effort was made to limit the modification to changes resulting from the guideline variance, however, it was unavoidable that global differences in the meshes resulted. A total of 8 modifications to the HLPW3 medium level mesh were generated as a result. The modified meshes were generated with the Pointwise ${ }^{\circledR}$ mesh generator to maintain consistency with the method used to generate the baseline mesh. A priori mesh metrics were presented for initial mesh comparison.

The impact of the guideline variances was assessed through comparison of node-centered, second-order, finite-volume, turbulent CFD simulations based on the resulting meshes. The flow simulations were computed with the NASA FUN3D CFD tool. The freestream conditions matched those of the HLPW3 "Grid Convergence Study" for the HL-CRM. Flow solution convergence was indicated by a standard deviation in $\mathrm{C}_{\mathrm{L}} \mathcal{O}\left(10^{-5}\right)$ over the final 1000 iteration steps.

With regard to $\mathrm{C}_{\mathrm{L}}$, all of the guideline variances could be considered reasonable if they reduce the time and resources to generate the mesh. However, when considering the prediction of velocity profiles, the Mod 3 variances would appear to be less desirable and should be avoided. This is due to the earlier termination of the boundary layer structures triggered by the increasing growth rate due to the added growth rate acceleration. As a result, there was less mesh resolution available to resolve and maintain the element wakes.

With regard to the computation of lift, it may be unnecessary to impose layers of constant cell height such as those dictated by Mod 2. Those guidelines are of concern to the accurate computation of skin friction and hence drag. As long as the error in drag is not significant to the point of altering lift, they may be unnecessary requirements for high lift simulation. This guideline proved to have little impact on either the $\mathrm{C}_{\mathrm{L}}$ or the extracted velocity profiles used in this assessment.

With respect to the node-centered, second-order, finite-volume, CFD solver FUN3D, it would appear that 5 points across the trailing edge is sufficient, as the deviation from the extracted velocity profiles with respect 


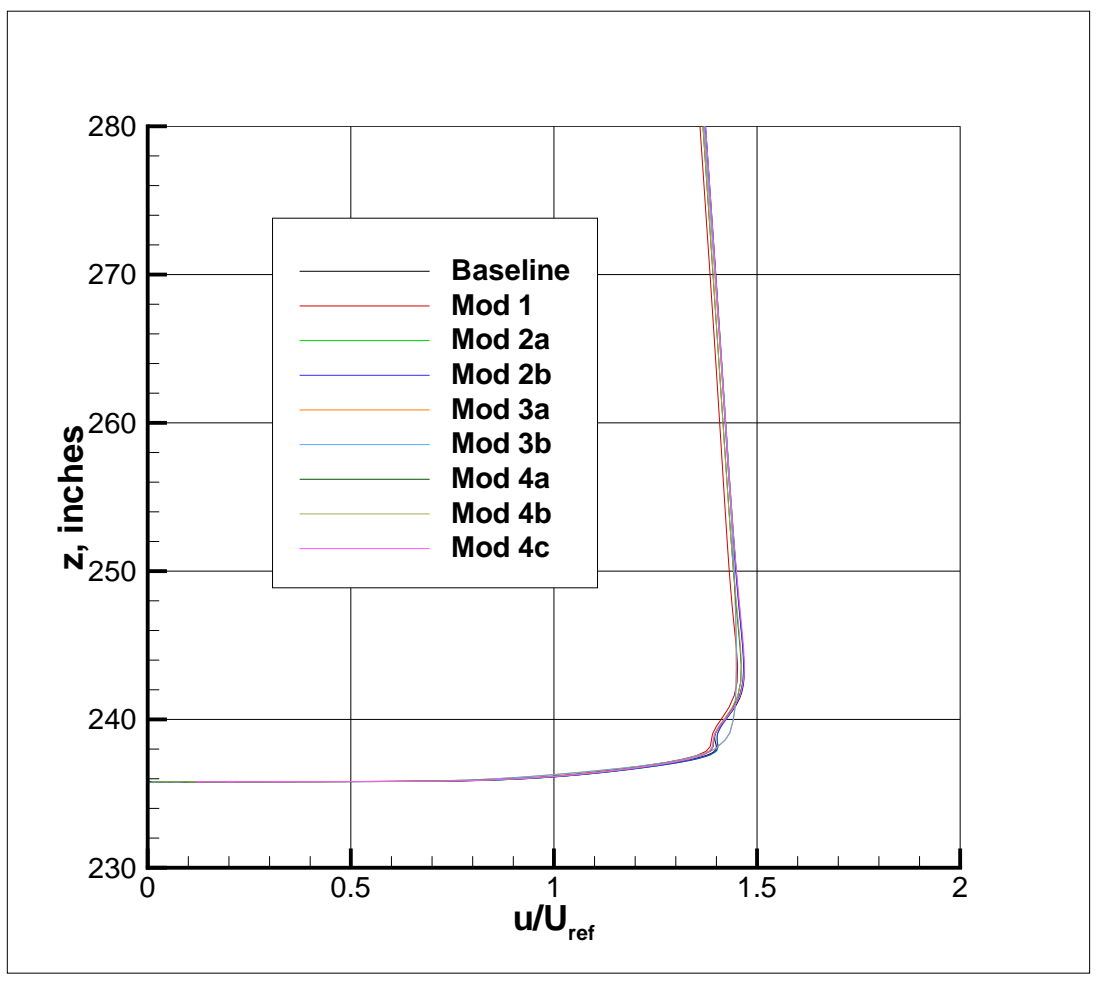

(a) Over the main element at $x=1495$ inches

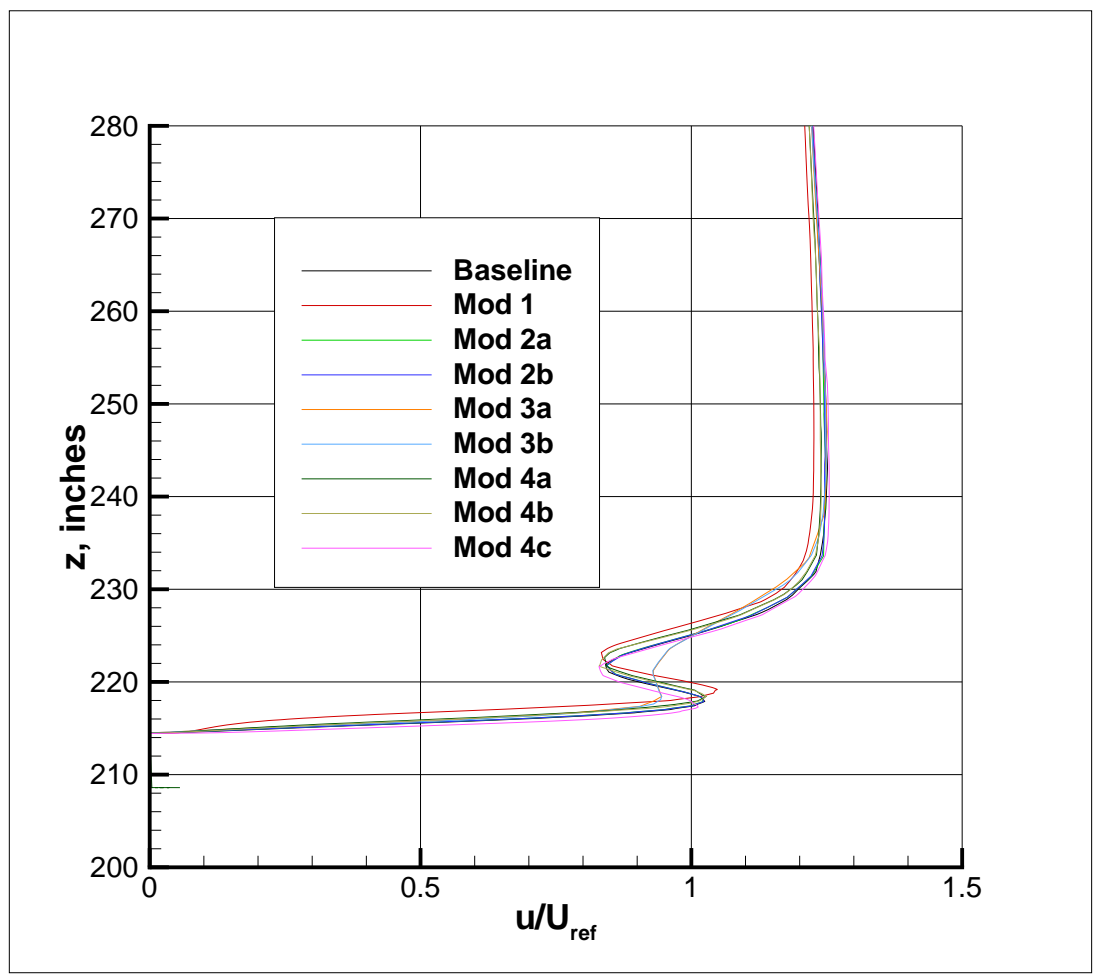

(b) Over the flap element at $x=1615$ inches

Figure 13. Velocity profiles at $y=638$ inches, $\alpha=8^{\circ}$. 


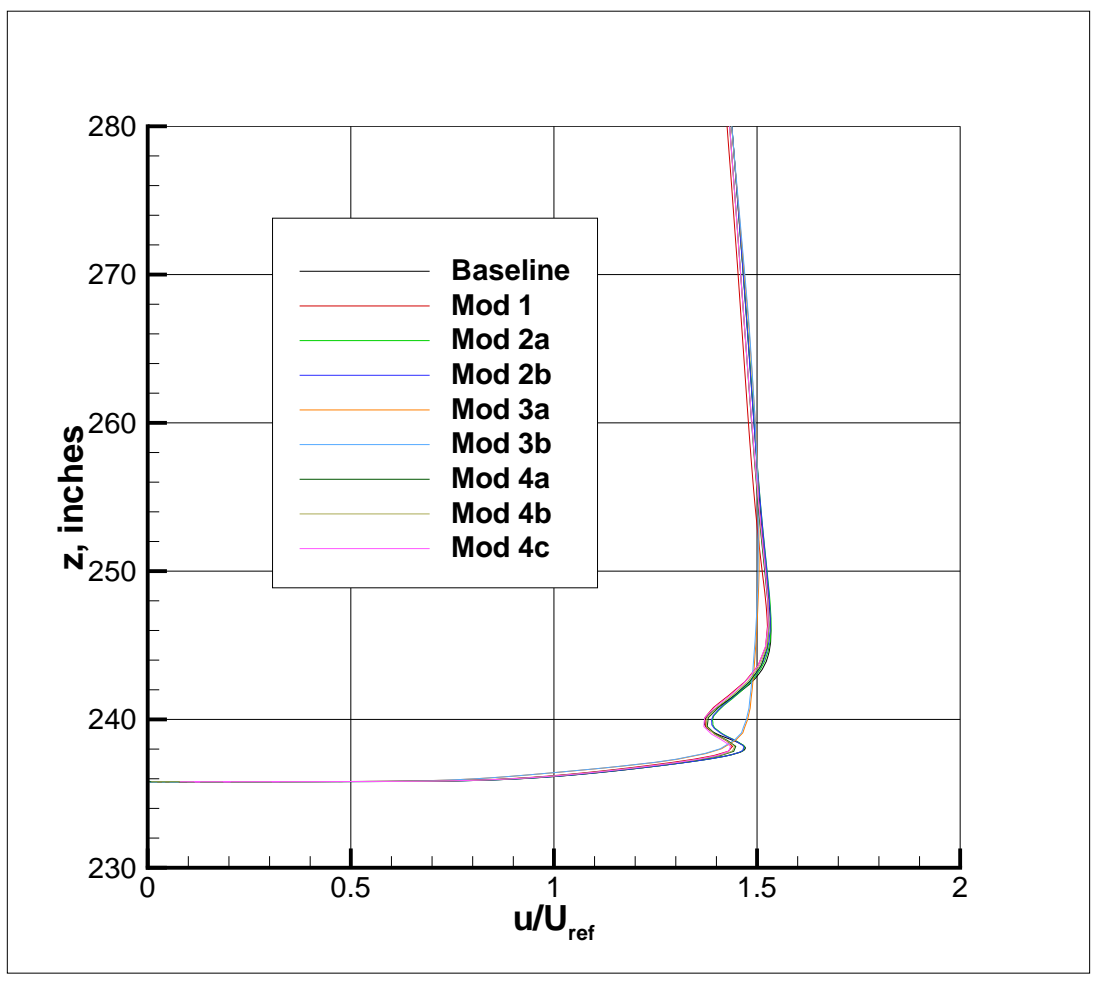

(a) Over the main element at $x=1495$ inches

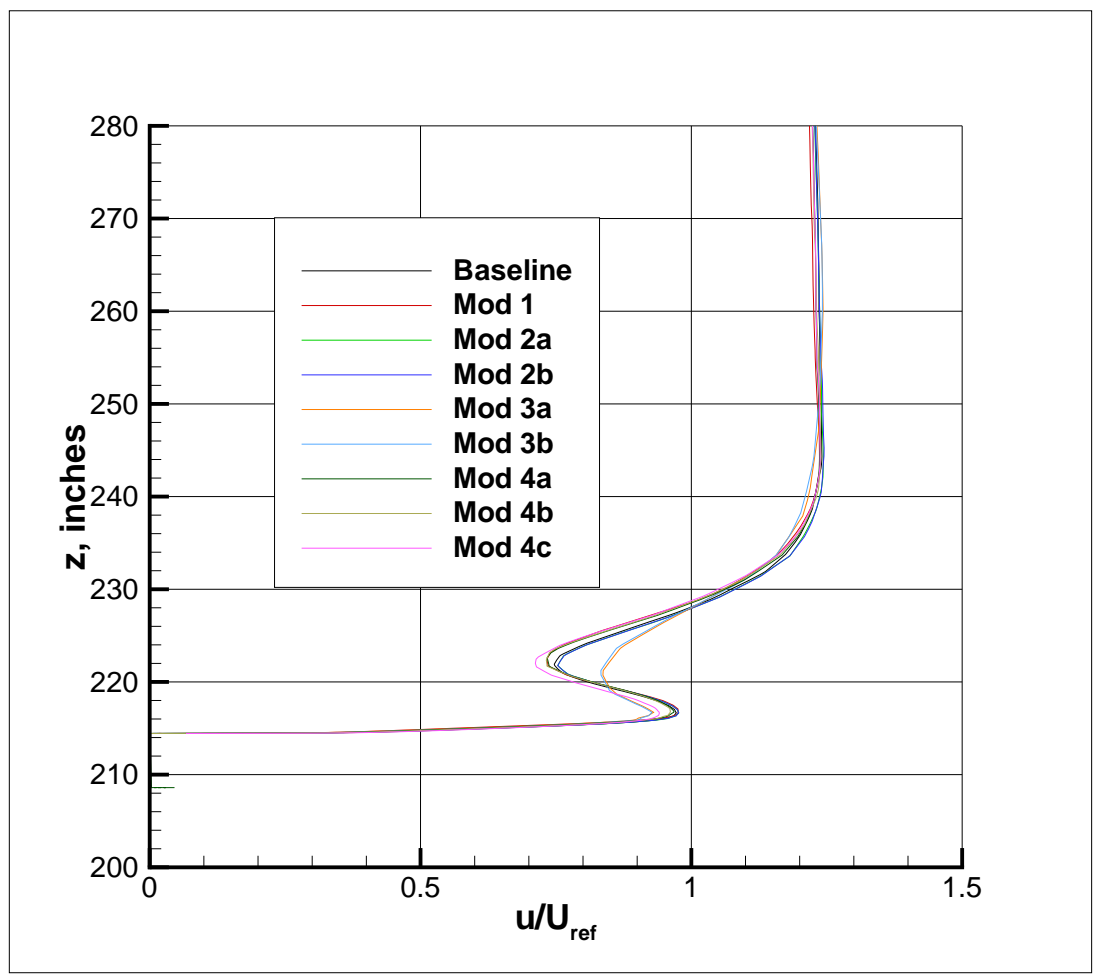

(b) Over the flap element at $x=1615$ inches

Figure 14. Velocity profiles at $y=638$ inches, $\alpha=16^{\circ}$. 


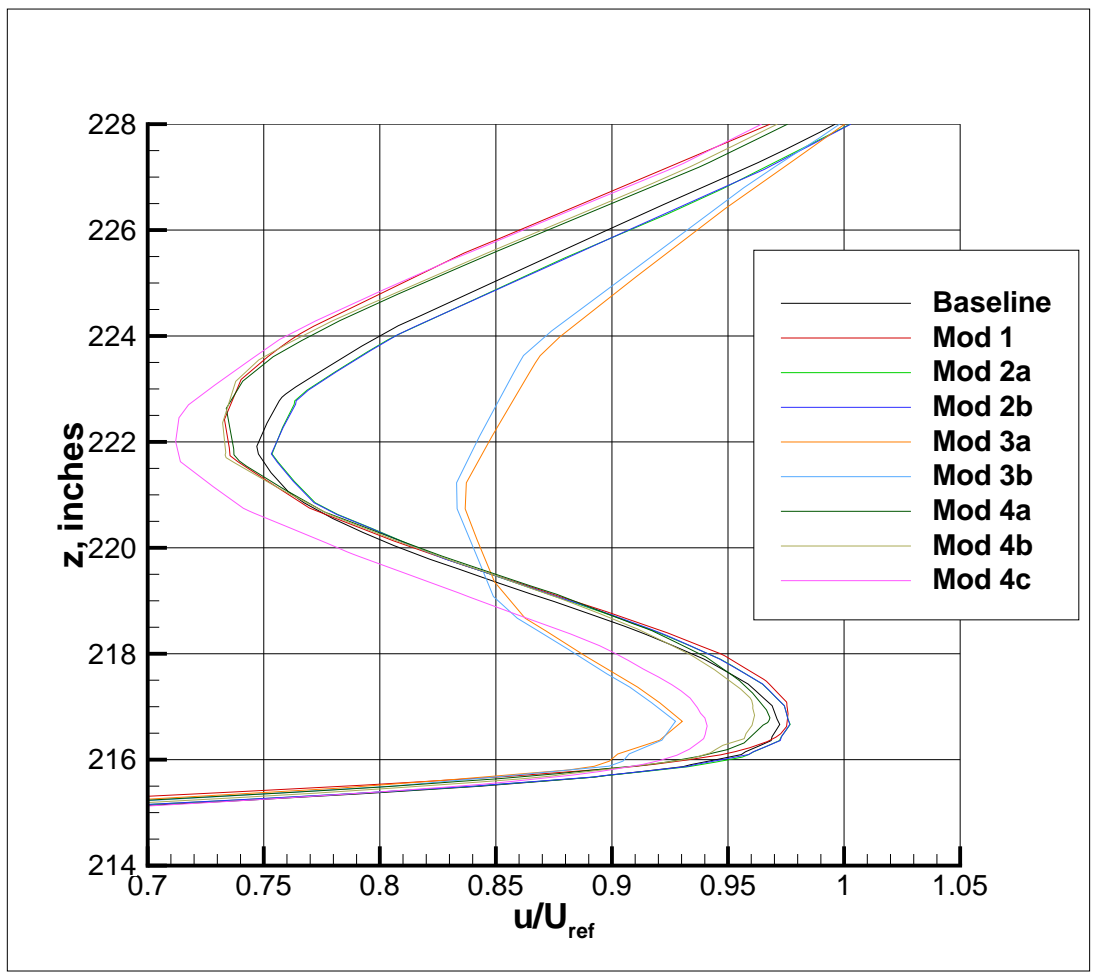

Figure 15. Detail of flap element velocity profiles at $x=1615$ and $y=638$ inches, $\alpha=16^{\circ}$.

to the case with 7 points (Mod $4 a$ ) and 9 points (baseline) is negligible.

Lastly, this study was conducted to assess the impact of meshing guideline variances on the accuracy of the computed flow solutions. It is left as a future exercise to determine the impact on productivity and practicality that results from augmentation and/or elimination of the established guidelines.

\section{Acknowledgments}

The authors would like to acknowledge the members of the $1^{\text {st }}$ Geometry and Mesh Generation Workshop and $3^{\text {rd }}$ High Lift Prediction Workshop committees for their hard work in organizing and conducting the workshops. Many thanks are owed to Dr. John P. Steinbrenner and Mr. Pat Baker of Pointwise for their work on the development of new meshing capabilities used within this study. Special thanks is also given to Dr. Christopher Rumsey of the NASA Langley Research Center for his suggestions toward assessment of the impact of the guideline variances. 


\section{References}

${ }^{1}$ Lacy, D. S. and Sclafani, A. J., "Development of the High Lift Common Research Model (HL-CRM): A Representative High Lift Configuration for Transonic Transports," AIAA Paper 2016-0308, San Diego, CA, Jan 2016.

2 " $3^{\text {rd }}$ AIAA CFD High Lift Prediction Workshop," URL: http://hiliftpw.larc.nasa.gov, June 2017, email: hiliftpw@gmail.com.

${ }^{3}$ Woeber, C. D., Gantt, E. J. S., and Wyman, N. J., "Mesh Generation for the NASA High Lift Common Research Model (HL-CRM)," AIAA Paper 2017-0363, Grapevine, TX, Jan 2017.

${ }^{4}$ Skaperdas, V. and Ashton, N., "Development of high-quality hybrid unstructured meshes for the GMGW-1 workshop using ANSA," AIAA Paper 2018-0660, Kissimmee, FL, Jan 2018.

${ }^{5}$ Langlois, M., Yang, H., and Sermeus, K., "Bombardier Contribution to the 3rd AIAA High-Lift Workshop," AIAA Paper 2018-1038, Kissimmee, FL, Jan 2018.

${ }^{6}$ Steinbrenner, J. P. and Abelanet, J. P., "Anisotropic Tetrahedral Meshing Based on Surface Deformation Techniques," AIAA Paper 2007-0554, Reno, NV, Jan 2007.

${ }^{7}$ Anderson, W. K. and Bonhaus, D. L., "An Implicit Upwind Algorithm for Computing Turbulent Flows on Unstructured Grids," Computers and Fluids, Vol. 23, No. 1, 1994, pp. 1-21, doi: 10.1016/0045-7930(94)90023-X.

${ }^{8}$ Anderson, W. K., Rausch, R. D., and Bonhaus, D. L., "Implicit/Multigrid Algorithms for Incompressible Turbulent Flows on Unstructured Grids," Journal of Computational Physics, Vol. 128, No. 2, 1996, pp. 391-408, doi: 10.1006/jcph.1996.0219.

${ }^{9}$ Alexandrov, N., Atkins, H. L., Bibb, K. L., Biedron, R. T., Gnoffo, P. A., Hammond, D. P., Jones, W. T., Kleb, W. L., Lee-Rausch, E. M., Nielsen, E. J., Park, M. A., Raman, V. V., Roberts, T. W., Thomas, J. L., Vatsa, V. N., Viken, S. A., White, J. A., , and Wood, W. A., "Team Software Development for Aerothermodynamic and Aerodynamic Analysis and Design," NASA TM-2003-212421, Nov 2003.

${ }^{10}$ Nielsen, E. J., Lu, J., Park, M. A., and Darmofal, D. L., "An Implicit, Exact Dual Adjoint Solution Method for Turbulent Flows on Unstructured Grids," Computers and Fluids, Vol. 33, No. 9, 2004, pp. 1131-1155, doi: 10.1016/j.compfluid.2003.09.005.

${ }^{11}$ Roe, P. L., "Approximate Riemann Solvers, Parameter Vectors, and Difference Schemes," Journal of Computational Physics, Vol. 43, No. 2, 1981, pp. 357-372, doi: 10.1016/0021-9991(81)90128-5.

${ }^{12}$ Spalart, P. R. and Allmaras, S. R., "A One-Equation Turbulence Model for Aerodynamic Flows," AIAA Paper 92-0439, Jan 1992.

${ }^{13}$ Rumsey, C. L., Slotnick, J. P., and Sclafani, A. J., "Overview and Summary of the Third AIAA High Lift Prediction Workshop," 2018, Manuscript submitted for publication.

${ }^{14}$ Nichols, R. H., Tramel, R. W., and Buning, P. G., "Solver and Turbulence Model Upgrades to OVERFLOW 2 for Unsteady and High-Speed Applications," AIAA Paper 2006-2824, San Francisco, CA, June 2006.

${ }^{15}$ Lawrence, S., "OVERFLOW: Facts on Friction," 1999 NASA High-Speed Research Program Aerodynamic Performance Workshop, Volume I-Configuration Aerodynamics, edited by D. E. Hahne and Ed., NASA CP-1999-209704/VOL1/PT1, 1999, pp. 401-416.

${ }^{16}$ Chan, W. M., Gomez III, R. J., Rogers, S. E., and Buning, P. G., "Best Practices In Overset Grid Generation," AIAA Paper 2002-3191, St. Louis, MO, June 2002. 